\title{
Extending the definition of quality for non-tunnelled central venous catheter insertion
}

\author{
Authors: Scott Oliver, Peter Thomson and Alan Jardine
}

\begin{abstract}
Aims
To improve the quality and safety of non-tunnelled central venous catheter (NTCVC) insertion in our tertiary-care renal unit, by extending traditional staphylococcal bacteraemia (SAB) surveillance to include additional markers of quality and risk.
\end{abstract}

\section{Methods}

Two experienced clinicians developed a series of potential indicators of risk or quality with respect to NTCVC insertion. Prospectively collected data were retrieved from our electronic patient record system, and analysed with respect to these variables. Data analysis was conducted using Microsoft Excel 2013.

\section{Results}

During the period 1 January 2013 - 31 December 2013, a total of 259 patients had 318 NTCVCs inserted, accounting for 1,038 NTCVC days. Most patients (220, 85\%) had one line, while 98 additional lines were inserted in 39 patients (range, two to eight lines per patient). NTCVC sites were internal jugular vein (179, $56.3 \%)$; femoral vein $(121,38 \%)$; subclavian vein $(1,0.3 \%)$. Site data were incomplete for 17 NTCVCs. Following NTCVC insertion, there were $12 \mathrm{SAB}$ episodes (11.08 per 1,000 NTCVC days); 15 patients $(4.7 \%)$ had $>=2 \mathrm{~g} / \mathrm{dL}$ drop in haemoglobin; and one pneumothorax $(0.3 \%)$ was reported on chest X-ray. Sixty-eight NTCVCs $(21.3 \%)$ were deemed inadequate as they were required to be replaced with a further NTCVC within 21 days of their insertion.

\section{Conclusions}

Despite SAB incidence significantly below Scottish and UK audit standards, our data demonstrated higher than expected proportions of femoral vein NTCVCs, and NTCVCs requiring replacement within 21 days. By expanding quality surveillance to include multiple variables rather than simply measuring $\mathrm{SAB}$ incidence, we have augmented our definition of quality and safety, and have illuminated new avenues for quality improvement.

\section{Conflict of interest statement}

No conflicts of interest to declare. 